\title{
A Similarity-based Inference Engine for Non-Singleton Fuzzy Logic Systems
}

\author{
Christian Wagner, Amir Pourabdollah, Josie McCulloch, Robert John and Jonathan M. Garibaldi \\ Lab for Uncertainty in Data and Decision Making (LUCID), School of Computer Science, \\ University of Nottingham, Nottingham, NG8 1BB, UK \\ Email: \{christian.wagner, amir.pourabdollah, psxjm5, robert.john, jon.garibaldi\}@nottingham.ac.uk
}

\begin{abstract}
In non-singleton fuzzy logic systems (NSFLSs) input uncertainties are modelled with input fuzzy sets in order to capture input uncertainty such as sensor noise. The performance of NSFLSs in handling such uncertainties depends both on the actual input fuzzy sets (and their inherent model of uncertainty) and on the way that they affect the inference process. This paper proposes a novel type of NSFLS by replacing the compositionbased inference method of type-1 fuzzy relations with a similaritybased inference method that makes NSFLSs more sensitive to changes in the input's uncertainty characteristics. The proposed approach is based on using the Jaccard ratio to measure the similarity between input and antecedent fuzzy sets, then using the measured similarity to determine the firing strength of each individual fuzzy rule. The standard and novel approaches to NSFLSs are experimentally compared for the well-known problems of Mackey-Glass time series predictions, where the NSFLS's inputs have been perturbed with different levels of Gaussian noise. The experiments are repeated for system training under both noisy and noise-free conditions. Analyses of the results show that the new method outperforms the standard approach by significantly reducing the prediction errors.
\end{abstract}

Index Terms-non-singleton, fuzzy logic systems, uncertainty, fuzzifier, input, similarity, time series prediction

\section{INTRODUCTION}

$\mathbf{F}$ UZZY Logic Systems (FLSs) have shown their effectiveness in a wide range of applications including engineering, natural science and time-series prediction [1], [2], [3]. Handling uncertain and vague information has been at the forefront of FLSs since the introduction of fuzzy sets (FSs) by Zadeh in 1965 [4]. Using Non-Singleton FLSs (NSFLSs) [5] allows the handling of the uncertainties associated with the inputs by modelling the inputs as input FSs [6]. For example, a FS with a Membership Function (MF) around $x$ (e.g., a Gaussian distribution centred on $x$ or a sensor-specific MF [7]) is used as the input FS [8]. In fact, SFLSs are a special case of NSFLSs where the input is represented by a singleton FS (i.e., a FS with a membership of 1 at $x$ and 0 elsewhere).

Although the theory of NSFLSs has been established for many years (e.g., in [5]), and the capacity of NSFLSs to deliver superior performance in comparison to SLFSs has been shown repeatedly [9], [10], the application of NSFLSs is still rare in comparison to SFLSs. The commonly cited reason for this is the additional computational and design complexity of NSFLSs compared to SFLSs [9], [10].

One way of interpreting the low use of NSFLSs is that the balance between performance gain and additional complexity has not been sufficient to warrant the additional complexity of their implementation as part of real world applications. Further, so far in the FLS community, research investigating the targeted modelling of individual sources of uncertainty (such as input uncertainty) for dedicated modelling in specific parts of the FLS (in this case - in the input FSs) has not been widespread, thus further limiting the appeal of NSFLSs which depend both on an accurate input uncertainty model and an effective mechanism to integrate the input uncertainty into the inference process. Thus, with additional research into uncertainty modelling for individual uncertainty types (such as input uncertainty) and the further development of effective and computationally reasonable NSFLS inference approaches, the attractiveness and applicability of NSFLSs will increase.

In previous papers [11], [12], we examined applying a new composition method between two fuzzy sets referred to as the cen-min method (such that the centroid of the fuzzy set intersection between input and antecedent fuzzy sets is used for calculating each rule's firing strength instead of the intersection's maximum currently used in standard NSLFSs) and compared the results in a noisy time series prediction context. The results showed that the mean-squared error (MSE) between the NSFLS predictions and the actual time series values was reduced for the new approach by about 7 to 17 percent for the noisy Mackey-Glass time series, and by about 3 to 11 percent in the noisy Lorenz time series - over different noise/training conditions. Initial exploration of the computational complexity of the proposed approach also showed that, while formally more complex, in real world applications there is no significant difference between the cenmin NSFLS and standard NSFLS approaches.

In this paper, instead of altering the composition method, we examine replacing the whole composition-based inference component of an NSFLS with a similarity-based inference component. In this method, the firing strength of each rule is determined by the similarity between the input and antecedent fuzzy sets, rather than being determined by the standard composition of fuzzy relations. We then assess the performance of such a NSFLS in predicting the same noisy MackeyGlass time series as in the previous papers which serve as a benchmark. Note that standard NSFLS solutions have already been designed for noisy Mackey-Glass time series prediction (e.g., in [5], [6]) and have been discussed and compared to in [11], [12].

The structure of this paper is as follows: First, we review the 


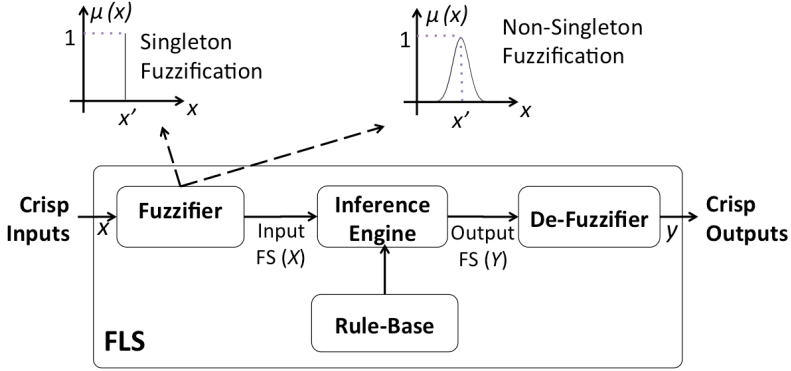

Fig. 1. The illustration of a typical FLS [12]. The fuzzifier component models the input as a fuzzy set with a membership function $\mu(x)$. Where the crisp input $x^{\prime}$ is certain, $\mu(x)$ can be a singleton FS (i.e., as in SFLS), or it can be a non-singleton FS where there is uncertainty about the crisp input $x^{\prime}$ (i.e. as in NSFLS).

background and the related works on NSFLSs, compositionbased and similarity-based inference methods. We proceed by proposing the new inference method for NSFLSs. In Section IV, we experimentally compare new and standard inference methods in NSFLSs using the application of noisy MackeyGlass time-series prediction. Finally, we conclude and provide directions for future work in Section VI.

\section{BACKGROUND}

In this section, we briefly introduce background material, including standard NSFLSs, the recently introduced centroidbased NSFLSs, similarity measures on FSs and existing developments around using such similarity-measures in the context of FLS inference.

\section{A. Non-Singleton FLSs}

Fig. 1 illustrates the building blocks of a NSFLS which employs a non-singleton fuzzifier for modelling the uncertainties embedded in the input. Here, in order to address uncertainty in the actual inputs, the fuzzifier maps a given crisp input to an input fuzzy set (FS), rather than to a fuzzy singleton as is the case in SFLSs. The appropriate type of membership function to be employed for the input FS is dependent on the characteristics of the input uncertainty, with the most common being a type of fuzzy number, i.e., a convex, normal FS [6]. In Fig. 1, a Gaussian distribution is shown as an example.

\section{B. NSFLSs with Standard Composition-based Inference}

The inference engine illustrated in Fig. 1 uses the different FSs from the fuzzifier and rule-base components (antecedent, and consequent FSs) in order to generate the output fuzzy set. For example, for Mamdani SFLS inference ([13]), the firing strength of each rule depends on the firing degree of each antecedent MF for each crisp (singleton FS) input. In NSFLSs, the computation is more complex since each antecedent has to be combined with the non-singleton input FSs in order for the firing strength of each rule to be determined. The details of the standard inference method for NSFLSs based on the composition of fuzzy relations are described in detail in [6], [14], including the mathematical detail on the relationship

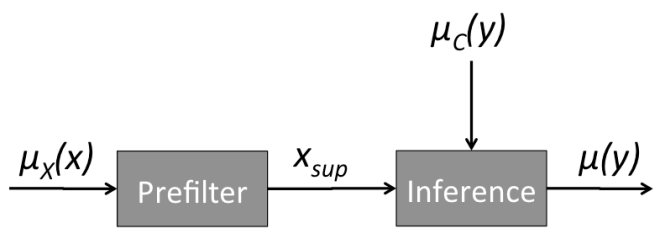

Fig. 2. Prefiltering of input FS to a NSFLS [12]

between inputs and outputs in such NSFLSs (i.e., between $X$ and output set $Y$ in Fig. 1).

We do not repeat the detail of the standard NSFLS inputoutput mapping here and instead we focus on the results. At an abstract level, we consider a single-input, single-rule and single-output system of Fig. 1 where Mamdani implication is used. Let $x$ and $y$ be members of input and output FSs $(X$, $Y$ ) and let $A$ and $C$ be two FSs representing an antecedent and a consequent. The only defined rule is If $x$ is $A$ then $y$ is $C$. We also define $\mu_{X}(x), \mu_{A}(x), \mu_{C}(y)$ and $\mu_{Y}(y)$ as the MFs of $X, A, C$ and $Y$ respectively. We notice that the output set of a one-rule inference engine is a composition between its input FS and a FS that is determined by a rule (each rule is considered as a fuzzy relation between antecedent(s) and a consequent, i.e., between the input space and output space. It can be shown that the input-to-output mapping of such a system is formulated as

$$
\mu_{Y}(y)=\mu_{C}(y) \star \mu_{X}\left(x_{\mathrm{sup}}\right) \star \mu_{A}\left(x_{\mathrm{sup}}\right),
$$

where $x_{\text {sup }}$ is the value of $x$ at which $\mu_{X}(x) \star \mu_{A}(x)$ takes its supremum. This equation is a reduced version of the general input-to-output mapping derived in [6] for the described abstracted NSFLS. It is also a direct result of employing supstar composition for fuzzy relations - as described in [6], [14]. Such an inference engine in a NSFLS can thus be imagined as a pre-filter unit [6] added to an inference unit of a SFLS, in which the pre-filter unit transforms the uncertain input set to a representative numerical value $x_{\text {sup }}$ (Fig. 2). Handling the input uncertainty in NSFLSs is concentrated in the prefiltering unit, i.e., the rest of the FLS acts identically to a singleton FLS.

In discrete systems (including the majority of FLS applications) the supremum operator is replaced by the maximum operator and $x_{\text {sup }}$ in equation (1) is replaced by $x_{\max }$. The most commonly used forms of sup-star composition in discrete systems are max-min and max-product compositions. In this paper we refer on the max-min composition, so $\mu_{X}(x) \star \mu_{A}(x)$ is the intersection of $X$ and $A$, and $\mu_{X}\left(x_{\max }\right)$ and $\mu_{A}\left(x_{\max }\right)$ are equal. As such, (1) can be written as

$$
\mu_{Y}(y)=\min \left[\mu_{A}\left(x_{\max }\right), \mu_{C}(y)\right] .
$$

Briefly, this formula tells us that the firing level of an antecedent is the peak of its intersection with the input set (Fig. 3). In a general case, the same formulation is iterated for an arbitrary number of inputs and rules.

We have briefly reviewed the standard inference method in NSFLSs based on sup-star (or specifically, the max-min) composition. Selecting $x_{\text {sup }}$ (or $x_{\max }$ ) to be the output of prefiltering is however clearly not the only possible method. 


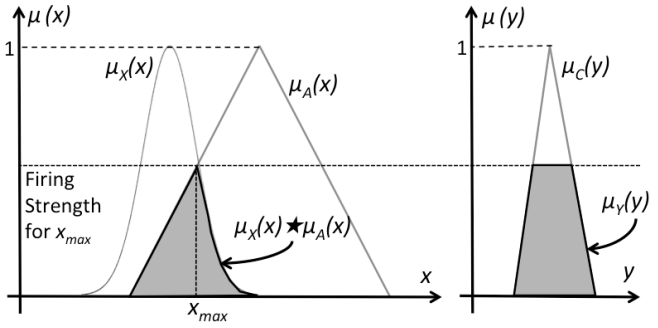

Fig. 3. The illustration of how a standard NSFLS calculates its output $(Y)$ according to its input $(X)$, antecedent $(A)$ and consequent $(C)$ FSs [12].

In the next sub-section, a previously introduced alternative to the standard composition method is briefly reviewed which has been shown to provide an improved capturing of the uncertainty modelled in input FSs.

\section{NSFLSS with Non-Standard Composition-based Inference}

The motivation for exploring potential variations to the standard sup-star composition methods was to identify approaches that can capture the interaction of input and antecedent FSs more accurately. Achieving this may provide a pathway for the uncertainty models of the inputs to be integrated in the overall FLS inference process with higher fidelity than possible in standard NSFLS thus far. The latter is vital both in terms of enabling better performance for NSFLSs where information on the uncertainty of the inputs is known, and to advance the wider ambition of directly modelling individual aspects of uncertainty where they occur (i.e., model input uncertainty in the input FSs).

In [11], [12], we presented a non-standard method which we refer to as the cen-NSFLS method which produced superior results compared to the standard NSFLS method. In cen-min composition method, the centroid of the intersection between the input and antecedent fuzzy set is used to determine each rule's firing level. The selection of the centroid is based on the fact that the shape of the intersection between the two FSs can represent more information about the interaction of the FSs, rather than the maximum or their intersection.

For example, in Fig 4, two different input FSs in a typical NSFLS are intersected with a single antecedent. Even though the actual input FSs are different, the firing levels calculated as part of the standard NSFLS method are the same in both cases. However, for the cen-min NSFLS method, the centroids of their intersections are different, so the cen-min method can produce different firing strengths, which better reflect the different intersections of the respective input and antecedent FSs.

In the initial experiments conducted, we showed that cenNSFLSs can provide a better performance in predicting both Mackey-Glass and Lorenz noisy time series (between 7\% to $17 \%$ reduced predicting MSE) compared to the standard max-min based NSFLS method, whereas the computation complexity overhead is negligible in practice [11], [12].

Besides the non-standard composition method, it is also possible to build the inference component based on a noncomposition method, namely based on measuring the similarity between the FSs. In the next sub-section, we briefly

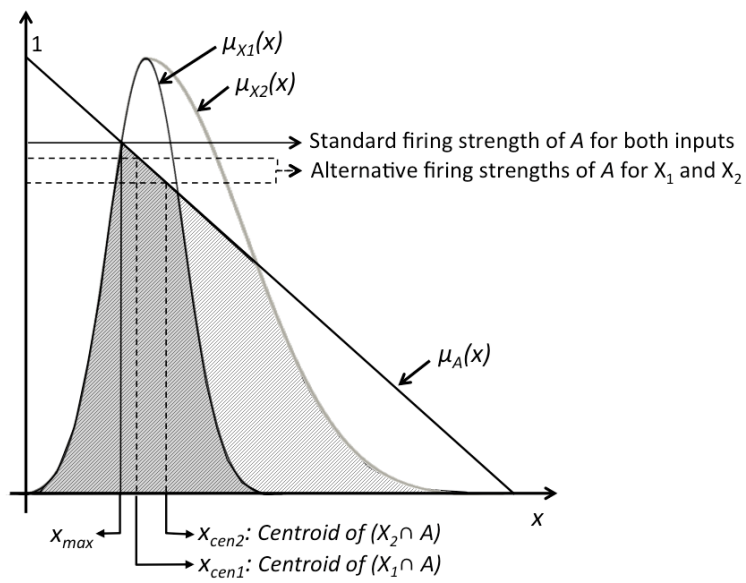

Fig. 4. The intersection of two different input fuzzy sets $X_{1}$ and $X_{2}$ with a single antecedent fuzzy set $A$, where $x_{\max }$ (and consequently the firing strength) is equal for both cases. Alternative firing strengths of $A$ are shown when the centroid of each intersection is used instead of their maximum [12].

review existing approaches to similarity-based inference and its applications.

\section{Similarity of Fuzzy Sets and Similarity-based Inference}

Similarity measures are often used on fuzzy sets to determine how closely two fuzzy sets share the same membership values across the universe of discourse. A similarity measure is a function $s: A \times B \rightarrow[0,1]$, where $A$ and $B$ are both fuzzy sets of the same type. A considerable number of similarity measures have been developed over the years. An overview of the core methods for computing similarity can be found in [15] and [16]. The properties of a similarity measure depend on the method taken and the context in which it is used, however, the most common properties used in the literature, which are also used within this paper, are:

Reflexivity:

$$
s(A, B)=1 \Longleftrightarrow A=B
$$

Symmetry:

$$
s(A, B)=s(B, A)
$$

Overlapping:

If $A \cap B \neq \emptyset$, then $s(A, B)>0$; otherwise, $s(A, B)=0$ Transitivity:

$$
\text { If } A \subseteq B \subseteq C \text {, then } s(A, B) \geq s(A, C)
$$

One of the most common similarity measures on fuzzy sets is the Jaccard ratio [17]. For two FSs $A$ and $B$ this is

$$
s(A, B)=\frac{\int_{x \in X} \min \left(\mu_{A}(x), \mu_{B}(x)\right)}{\int_{x \in X} \max \left(\mu_{A}(x), \mu_{B}(x)\right)} .
$$

This has all of the properties listed above.

One application of similarity on fuzzy sets strongly similar to its application considered in this paper is similarity based inference. The latter has also been referred to as fuzzy analogical reasoning and has been applied for many year in (fuzz) expert systems. It is a method of reasoning in which the consequent of a rule is modified according to the similarity between the input and the antecedent [16]. For example, Turksen and Zhong [18] use a proximity based similarity measure to compare 
the input with each rule antecedent. Here, if the resulting similarity is greater than a given threshold then the rule is fired. The consequent is then modified according to the degree of similarity, essentially resulting in a fuzzy hedge of the original consequent. It is noteworthy that this established approach to reasoning shares the fundamental methodology of leveraging the similarity between input and antecedent FSs with the approach proposed in this paper. The main difference is that in this paper, we focus on developing this mechanism as an alternative approach to standard NSFLSs, contrasting it to both the standard NSFLS approach and the recently introduced cenNSFLS appraoch as introduced in Section II-C. The motivation also differs, where this paper aims to establish the foundation for a new branch of research around FLSs, where a priori knowledge on different types of uncertainty (e.g., uncertainty affecting the inputs in this case) is addressed by and designed for in specific parts of FLSs (e.g., the input FSs).

Yeung and Tsang [19] also developed an approach similar to that in [18] using inclusion and cardinality to determine if a rule should be fired. The resulting value is used to modify the consequent fuzzy set in a similar manner to Turksen and Zhong's approach [18]. Additionally, Wang et al. [20] developed a method of inference using a fuzzy similarity measure, and Yeung et al. [21] used subsethood instead of similarity to determine if a rule should be fired.

In the next section, we present how the described fuzzy similarity measure may be utilized in NSFLSs.

\section{SIMILARITY-BASED INFERENCE FOR NSFLSS}

In this section we investigate the benefits of utilizing similarity-based inference for NSFLSs as an alternative to composition-based inference methods. Specifically compared to the standard composition-based inference, we highlight that selecting $x_{\text {sup }}$ may lead to the loss of valuable information encoded in the input FS membership function (which captures the uncertainty of the FLS input), thus an alternative inference method based on Jaccard similarity will be provided.

\section{A. Motivation}

Fuzzy systems are credited widely with dealing well with uncertainty in systems. However, exactly how to leverage this capacity in the best way possible based on insight on real applications (and uncertainties present) is still not well understood. Providing mechanisms which clearly and comprehensively link the uncertainty captured by input fuzzy sets to the antecedents and thus performance of the fuzzy system is a vital step to advancing the research around fuzzy systems as a whole.

In turn, this should enable the separation of input uncertainty and linguistic antecedent uncertainty modelling, both of which are commonly "mixed" in antecedent FSs in the case of singleton FLSs. We feel that this separation of uncertainty based on its origin is valuable and important, in particular as it enables the capture and modelling of uncertainties where they arise, i.e., separately in the inputs (e.g., a sensor) or in the antecedents (e.g., experts are unsure about the linguistic labels - what is "low"). The latter is vital to pave the way

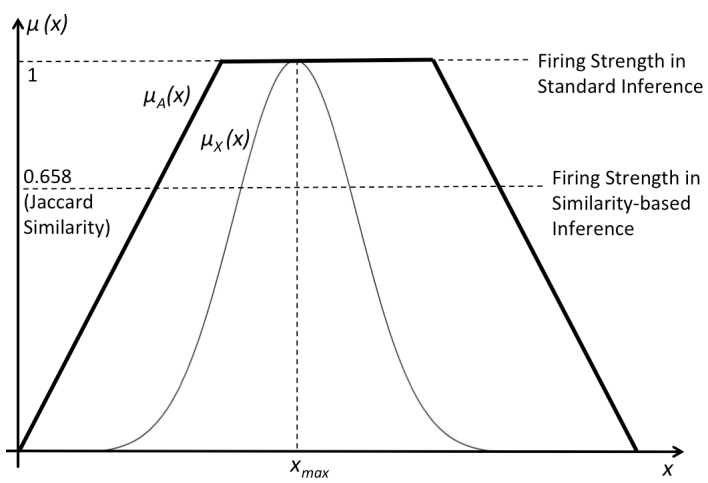

Fig. 5. The interaction of an input FSs $X$ with a single antecedent FS $A$ is shown. Note that the firing strengths for the standard NSFLS approach is equal to one, even though the input FS is very different to the actual antecedent FS. In similarity-based inference however, the firing strengths is equal to the FSs' similarity (here: 0.658 based on Jaccard similarity).

for the development of improved, systematic design methods for real world fuzzy systems and the eventual much wider applicability and easier deployability of fuzzy systems.

In the previous section, we highlighted that the pre-filtering stage is the critical step in the functionality of NSFLS's when standard composition is used. Specifically, this is the stage is where for each rule, the sup-star composition is used to combine the information encoded in the input set(s) with that of the antecedent set(s) to generate the firing level of the rule. In this paper we are particularly interested in establishing an efficient method that can perform the combination between input and antecedent sets as comprehensively as possible - minimising information loss about the distribution of uncertainty in the input fuzzy sets' membership functions.

To illustrate, we provide an example in Fig. 5. An input FS (shown with a Gaussian membership function) is interacting with an antecedent with a trapezoidal membership function. It is intuitive that a reasoning based on a rule "IF $X$ is $A$ THEN..." should not be fully fired unless the $\mathrm{X}$ and $\mathrm{A}$ are identical. However in this example, the rule is fully fired within the standard max-min method, since the two FSs eventually have a common maximum where $\mu_{X 1}\left(x_{\max }\right)=\mu_{X 2}\left(x_{\max }\right)=1$. This could happen with any other antecedent with a different shape if it has a common maximum with the input FS. A different example showing potential shortcomings of the standard NSFLS approach in contrast to the previously introduced cen-NSFLS approach was shown in Fig. 4. However, even for a cen-NSFLS (See Section II-C), it is possible that non-similar input and antecedent FSs produce high firing strengths simply because their intersection may have high membership grades at their centroids, rather than because the input FS actually strongly matching the antecedent FS. In other words, composition-based NSFLS approaches all have the potential pitfall that the firing strength of a given rule may not reflect the desired interaction between well-specified input and antecedent FSs.

Based on the above, it is arguable that a compositionbased inference method may not necessarily be the best choice in terms of capturing the interaction between input and antecedent FSs with the highest fidelity possible. Consequently, 
it seems desirable to identify an approach which results in a better capture of the detailed interaction of the input and antecedent set, thus resulting in a more appropriate firing level. Intuitively, measuring the similarity between the shapes of the input and the antecedent FSs can provide a suitable alternative to composition-based approaches.

\section{B. The Similarity-based Inference for NSFLSS}

Note that the similarity measures incl. the Jaccard similarity measure (see Section II-D) represent similarity by definition as a number in $[0,1]$. This conveniently enables the direct application of the similarity as a degree of firing for a given pair of input and antecedent FS.

To recapitulate from 3 , for $X$ and $A$ representing the input and antecedent FSs in the single rule IF X IS A THEN $Y$ is $C$, then their interaction based on the Jaccard similarity ratio is:

$$
s(X, A)=\frac{\int_{x \in X} \min \left(\mu_{X}(x), \mu_{A}(x)\right)}{\int_{x \in X} \max \left(\mu_{X}(x), \mu_{A}(x)\right)} .
$$

For input-output mapping within the rule, we employ minimum as the standard t-norm. Thus, if this is the only rule of the rule-base, the output fuzzy set will be:

$$
\mu_{Y}(y)=\min \left[s(X, A), \mu_{C}(y)\right]
$$

For multiple-input and multiple-rule system, the same extension used in the standard NSFLS method ([5]) can be used. We refer to this alternative NSFLS approach as sim-NSFLS hereafter (whereas the standard-NSFLS and cen-NSFLS were introduced in Section II). The following section focuses on the exploration of this new version of NSFLS through a series of experiments and result analysis.

\section{EXPERIMENTS AND RESULTS}

Following the detailing of the similarity-based inference for NSFLSs, we design both a standard-NSFLS and a simNSFLSs for time-series predicition, specifically, for the the prediction of the Mackey-Glass (M-G) chaotic time series under different Gaussian noise conditions (reflected in the different input FSs), and compare the results. In addition, we compare both types of NSFLSs to the previously introduced cen-NSFLS method ([11], [12]).

In the next subsections, firstly the experimental setting is explained, after which the results will be discussed.

\section{A. The Time Series Experiment}

The method described in [5] uses standard NSFLSs for the prediction of M-G time series in noisy conditions and compares the results to SFLSs. We follow a similar approach but conduct the same experiment using bpth standard and a similarity-based NSFLS, as well as a SFLS. We use the MSE (Mean Square Error) for estimating the deviation of the prediction from the expected time-series value.

The M-G time series [22] uses the following differential equation for building the next samples out of the previous samples:

$$
\frac{d x(t)}{d x}=\frac{0.2 x(t-\tau)}{1+x^{10}(t-\tau)}-0.1 x(t)
$$

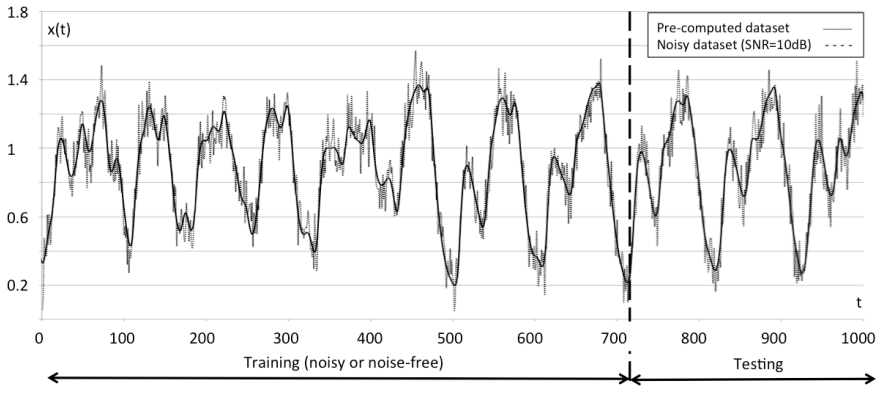

Fig. 6. The illustration of the pre-computed (noise-free) and noisy (Gaussian, $\mathrm{SNR}=10 \mathrm{~dB}$ ) time series [12]. The system is trained from $t=1$ to $t=700$ and tested from $t=701$ to $t=1000$. The period $t=-999$ to $t=0$ (not shown here) is for the initial transients to die out.

For $\tau>17$, (6) demonstrates chaotic behaviour. We have selected $\tau=30$. Using (6), $x(t)$ is calculated for 2000 consecutive time points, i.e., $t=-999$ to $t=1000$. The first 1000 points are for the initial transients to die out, then using points $t=1$ to $t=700$, the system is trained to develop its rule-base. The last 300 points from $t=701$ to $t=1000$ are used for testing the system. Fig. 6 shows the pre-computed and an example of a noisy time series for SNR (Signal to Noise Ratio) of $10 \mathrm{~dB}$ in training and testing intervals.

Rules are trained according to the well-known WangMendel one-pass method described in [23]. Nine past points in the time series are employed as inputs to generate a predicted value. Seven equally-distributed triangular MFs are used to model the antecedent. Both noisy and noise-free samples can be used for the rule generation process. We explored both of them to reflect both real world and noise free (lab-) conditions. In noise-free training, the same rule-base generated for the SFLS is used for all FLSs whereas in noisy training the rulebase is re-trained with noisy inputs.

Three FLSs are designed: a SFLS, a standard NSFLS and a sim-NSFLS. For all the systems, centroid deffuzification and Mamdani inference is used with the min and max operators for the $\mathrm{t}$-norm and $\mathrm{t}$-conorm respectively. The same discretization level (100 steps) is used for all FLSs. The input FS models used for both NSFLSs are Gaussian MFs centred on the crisp (noise-free) inputs, with a standard-deviation equal to that of the noise added in the given experiment (Fig. 7). Note that this approach to adjusting the input FSs to respective noise levels is a simplistic approach to designing for the levels of uncertainty/noise expected which is sufficient for the purpose of this paper. However, in the future we expect that improved approaches to modelling input FSs for a priori known uncertainty levels will be identified. For both NSFLSs, two experiments are conducted for lower and higher signalto-noise ratios (SNRs), namely for $10 \mathrm{~dB}$ and $5 \mathrm{~dB}$.

Each experiment generates 300 outputs which are compared to the pre-computed outputs calculated by (6). The MSE over the 300 points is used as a measure of the overall error for a given FLS. In order to mitigate the effect of randomness, each experiment is repeated 30 times and the generated MSEs are averaged. The next sub-section provides the experiments' results. 


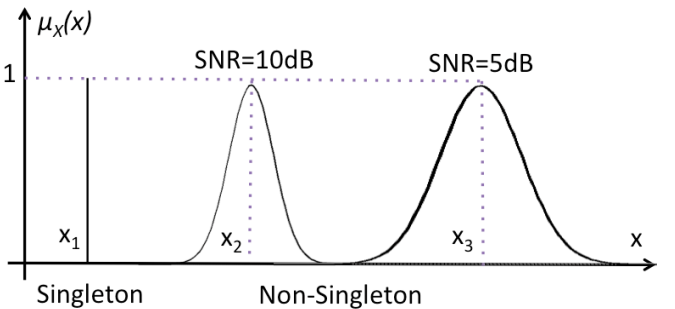

Fig. 7. Three different uncertainty models used in the experiments [12]. $x_{1}$, $x_{2}$ and $x_{3}$ represent three actual inputs to the system where their uncertainties are modelled by three different fuzzification types.

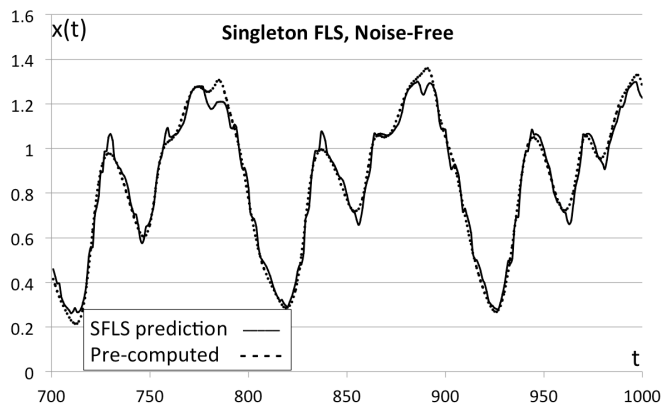

Fig. 8. FLS output with no noise in the inputs, when trained with noise-free data compared to the pre-computed dataset [12].

\section{B. Results}

First, we examine the system's functionality in a noise-free setting where there is no uncertainty in the inputs (i.e., a SFLS should provide perfectly adequate performance). The SFLS is trained for points $0<t \leq 700$ of the time series over which 184 rules are generated. The prediction results (Fig. 8), for $700<t \leq 1000$ show that the system is relatively well trained and closely follows the pre-computed ground-truth of the $\mathrm{M}-\mathrm{G}$ time series.

As a second step, we add noise to the inputs, while still using the same rule-base generated in the previou step, i.e. using noise-free training. This reflects the case where a system is trained in lab-conditions and tested in the (noisy) real-world. We subsequently compare the performance of the standardNSFLS with that of the proposed sim-NSFLS approach, where for each, the standard deviations of the Gaussian input FSs are adjusted to reflect the level of noise injected as explained above. The prediction results for two different noise levels are illustrated in Fig. 9.

Thirdly, we re-generate the rule-base using noisy samples (Gaussian noise on SNR=5dB, 10dB) from $0<t \leq 700$ and re-test the resulting FLSs for $700<t \leq 1000$. This reflects real-world training and application where noise is unavoidable at all stages. The Wang-Mendel approach generates 557 rules for $\mathrm{SNR}=10 \mathrm{~dB}$ and 664 rules for $\mathrm{SNR}=5 \mathrm{~dB}$. Fig. 10 shows the prediction results for the two different noise levels, for both the standard- and the sim-NSFLSs.

The performance results (based on the measured MSEs) are summarized in Table I. On average over all the experiments, the sim-NSFLS shows a reduced deviation from the precomputed time series and thus better performance in comparison to the standard NSFLS. Note that the difference in

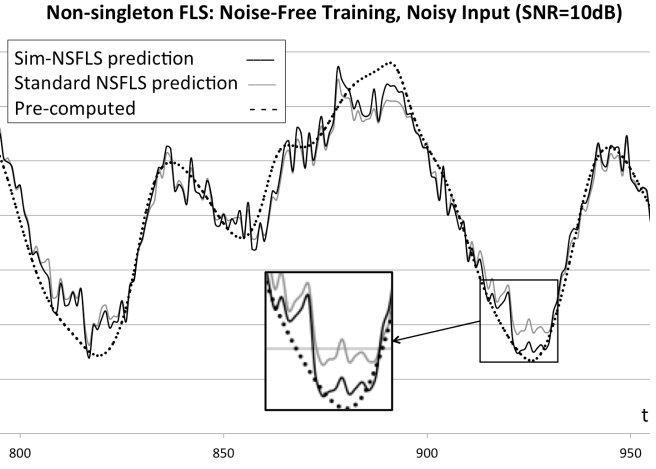

(a)

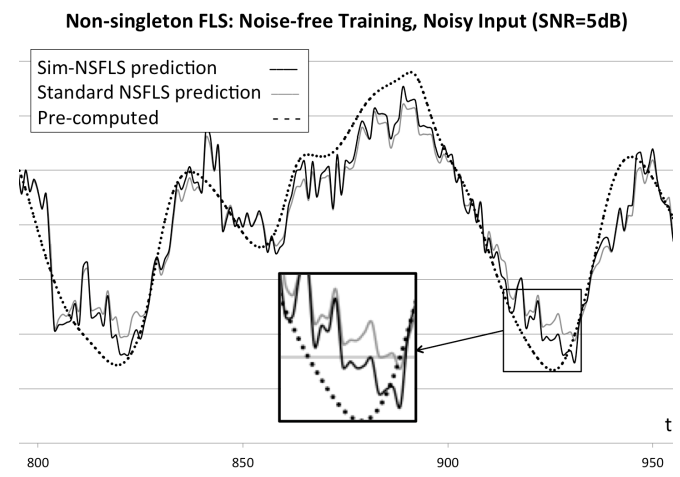

(b)

Fig. 9. (a) Comparing the standard and similarity-based NSFLS outputs when they are trained with noise-free data and tested with noisy data with $\mathrm{SNR}=10 \mathrm{~dB}$. (b) the same for $\mathrm{SNR}=5 \mathrm{~dB}$.

performance here originates only from the change in the actual NSFLS mechanism.

TABLE I

THE PREDICTION PERFORMANCE (FOR M-G TIME SERIES) PRODUCED BY THE DIFFERENT FLSS BASED ON AVERAGE MSES (MEAN SQUARE ERRORS).

\begin{tabular}{|l|l|l|l|l|l|}
\hline System & $\begin{array}{l}\text { SNR } \\
(\mathbf{d B})\end{array}$ & Training & $\begin{array}{l}\text { MSE } \\
\text { (Standard- } \\
\text { NSFLS) }\end{array}$ & $\begin{array}{l}\text { MSE } \\
\text { (Sim- } \\
\text { NSFLS) }\end{array}$ & $\begin{array}{l}\text { Change } \\
(\boldsymbol{\%})\end{array}$ \\
\hline SFLS & $\mathrm{n} / \mathrm{a}$ & n/a & 0.00138 & 0.00138 & 0.00 \\
\hline NSFLS & 10 & noise-free & 0.00673 & 0.00514 & -23.63 \\
\hline NSFLS & 5 & noise-free & 0.01546 & 0.01187 & -23.33 \\
\hline NSFLS & 10 & noisy & 0.01236 & 0.00889 & -28.15 \\
\hline NSFLS & 5 & noisy & 0.04016 & 0.02753 & -31.57 \\
\hline
\end{tabular}

\section{Discussion}

The provided results show that by changing from standardNSFLS to sim-NSFLS, the deviation of the predicted output from the expected output has dropped in all four NSFLS experiments. The highest improvement $(31.57 \%)$ is found for noisy training with higher levels of noise and the lowest improvements (around 23\%) is achieved when the training is done with noise-free samples. Note that the SFLS results are provided for reference only as no noise is present in these experiments.

Going beyond the comparison to the standard NSFLS approach, it is interesting to compare the provided results 
Non-singleton FLS: Noisy Training, Noisy Input (SNR=10dB)

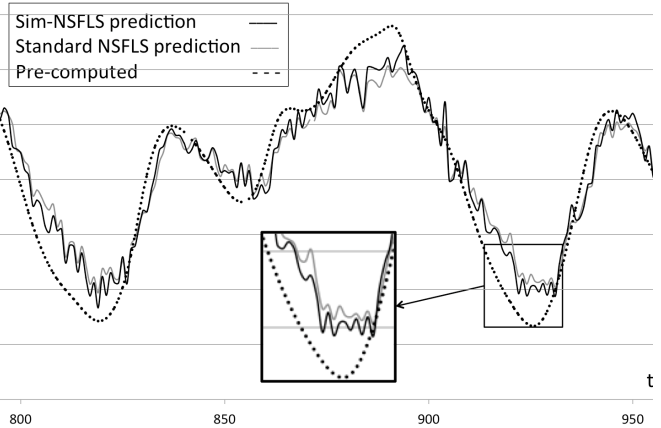

(a)

Non-singleton FLS: Noisy Training, Noisy Input (SNR=5dB)

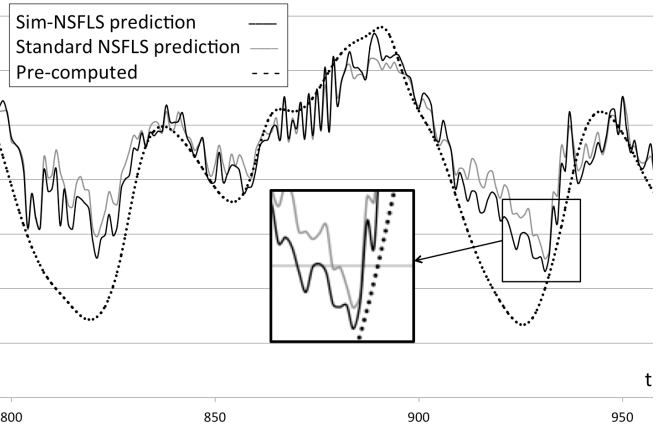

(b)

Fig. 10. (a) Comparing the standard and the similarity-based NSFLS outputs when they are trained and tested with noisy data with $\mathrm{SNR}=10 \mathrm{~dB}$. (b) the same for $\mathrm{SNR}=5 \mathrm{~dB}$.

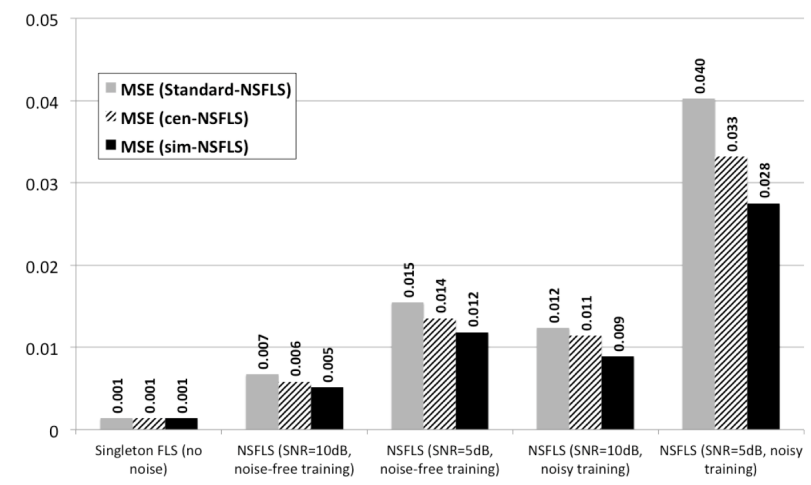

Fig. 11. Comparing the MSEs produced by standard-, cen- and sim-NSFLSs in predicting $\mathrm{M}-\mathrm{G}$ time series in different experiment settings.

with the results of the previously introduced cen-NSFLSs [12] approach. As shown in Fig. 11, the prediction performance of cen-NSFLS is better than that of the standard-NSFLS but lower than that of the sim-NSFLS in all the experiments. This result is intuitive as the cen-NSFLS provides a more fine-grained matching of input and antecedent FSs than the standard NSFLS approach but which is still less detailed than the similarity-based approach employed proposed for simNSFLSs.

Having a closer look at the results, for example in Figs. 9 and 10 , it is noticeable that although the prediction by the sim-NSFLS has improved for the majority of samples, this is not the case for all of the sample points. Specifically, in
TABLE II

THE NUMBER OF SAMPLES (OUT OF 300) WHERE EACH METHOD IS OUTPERFORMED IN DIFFERENT NSFLS CONFIGURATIONS

\begin{tabular}{|l|l|l|}
\hline & $\begin{array}{l}\text { sim-NSFLS } \\
\text { outperforming } \\
\text { standard-NSFLS }\end{array}$ & $\begin{array}{l}\text { cen-NSFLS } \\
\text { outperforming } \\
\text { standard-NSFLS }\end{array}$ \\
\hline $\begin{array}{l}\text { SNR=10dB } \\
\text { noise-free training }\end{array}$ & 200 & 216 \\
\hline $\begin{array}{l}\text { SNR=5dB } \\
\text { noise-free training }\end{array}$ & 209 & 214 \\
\hline $\begin{array}{l}\text { SNR=10dB } \\
\text { noisy training }\end{array}$ & 225 & 204 \\
\hline $\begin{array}{l}\text { SNR=5dB } \\
\text { noisy training }\end{array}$ & 195 & 216 \\
\hline
\end{tabular}

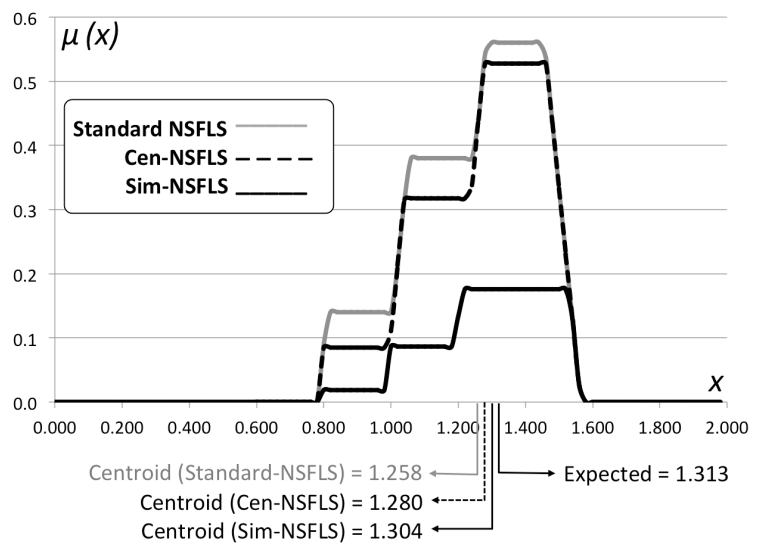

Fig. 12. For $\mathrm{t}=1000$, three output fuzzy sets produced by the standard-NSFLS, cen-NSFLS and sim-NSFLS are shown together with the centroid of each fuzzy set and the expected value.

a sample-by-sample analysis in an individual system running (from $\mathrm{t}=701$ to $\mathrm{t}=1000$, non-noisy training and $\mathrm{SNR}=10 \mathrm{~dB}$ ), the sim-NSFLS outperformed the standard NSFLS in 200 out of 300 samples in our experiments. In order to enable the compariosn of this to the same analysis conducted for the cenNSFLS in relation to the standard-NSFLS on the same data samples in [12], Table II shows the number of times that each of the novel NSFLSs outperformed the standard one over the 300 samples for the different NSFLS settings.

Finally, in order to further detail the actual difference in behaviour between the different types of NSFLS, we choose an individual sample point where both the sim-NSFLS and the cen-NSFLS has outperformed the standard-NSFLS. Clearly, different points may be chosen - the selection is merely for demonstration purposes. Specifically, we chose the outputs FSs for sample $t=1000$ from the experiment with non-noisy training and $\mathrm{SNR}=10 \mathrm{~dB}$. For this case, the defuzzified outputs of the three systems (the standard, the cen-NSFLS and the sim-NSFLS) are 1.258, 1.280 and 1.304 respectively, whereas the expected value is 1.313 . The three predicted values are the result of the centroid defuzzification of the individual NSFLSs' output sets which have been illustrated in Fig. 12.

This particular set of results shows the better estimation of the actual value by the similarity-based NSFLS compared to the other two systems. It is noticeable that the membership grades of the output fuzzy set produced by the sim-NSFLS 
are generally the lowest (as can be expected for similarity) but result in the closest match to the expected value.

\section{CONCLUSIONS AND Future Work}

In this paper we proposed a similarity-based method of inference for non-singleton fuzzy logic systems in order to enable a higher fidelity capture of the input uncertainty embodied by input fuzzy sets in comparison to the standard composition method employed in NSFLSs. In the novel approach, the sup (supremum - commonly maximum) composition is replaced by the similarity-based inference, where the Jaccard similarity ratio is used to estimate how similar each input FS is to each fuzzy rule's antecedent FS. While the paper employed the Jaccard measure throughout, clearly other measures of similarity could be employed. The resulting similarity (a number between 0 and 1) is then used to determine the firing level of each rule within a Mamdani inference method.

A series of experiments was performed to test the performance of the newly proposed NSFLS in predicting $M-G$ time series, showing an improved prediction performance for the proposed approach. While these improvements in timeseries prediction are interesting in their own accord, the more fundamental outcome is that they indicate that the proposed approach has the potential to provide high-fidelity matching between noisy inputs and antecedents. In other words, while the current paper has not focussed on actually how to best design input FSs for the given levels of uncertainty, the proposed approach makes such work substantially easier and potentially rewarding, as accurate uncertainty models have an improved potential to directly result in improved performance. The latter is valuable for applications where the nature of the uncertainty is known a priori, but also for example facilitates learning and optimisation approaches where the fuzzification stage is tuned in response to different noise conditions.

Clearly however, the results and conclusions in this paper on the performance of the proposed NSFLS approach are solely based on a single, specific setting for the $\mathrm{M}-\mathrm{G}$ time series. Further work will be required to more generally evaluate the proposed approach, both in terms of performance/behaviour and in terms of computational complexity which is relevant in real-time applications such as fuzzy logic control. With this in mind, the scalability of the results to a wider range of NSFLS applications (both in and beyond time-series prediction) is a direction for our future work.

Further, comparing the proposed method with our previous work on a centroid-based NSFLS method shows that the new method has significantly improved the NSFLS performance beyond what was achieved previously. However, the results show that although the cen-NSFLS prediction error is higher than that of the sim-NSFLS overall (on a verage); it has outperformed the standard-NSFLS in more samples than the sim-NSFLS. We expect that this is related to the actual design of the input FSs, but a more detailed investigation will be conducted in the future.

Finally, clear other routes for further development of the work in this paper include the exploration of other fuzzy similarity and/or distance measures [24] in NSFLSs for comparing input and antecedent FSs, exploring different input fuzzy set generation methods in respect to uncertainty/noise, and extending the approach to type-2 NSFLSs.

\section{REFERENCES}

[1] G. Bojadziev and M. Bojadziev, Fuzzy logic for business, finance, and management. World Scientific Publishing Co., Inc., 2007.

[2] L. Duckstein et al., Fuzzy rule-based modeling with applications to geophysical, biological, and engineering systems. CRC press, 1995, vol. 8 .

[3] J. Yen, R. Langari, and L. A. Zadeh, Industrial applications of fuzzy logic and intelligent systems. IEEE Press, 1995.

[4] L. A. Zadeh, "Fuzzy sets," Information and control, vol. 8, no. 3, pp. 338-353, 1965.

[5] G. C. Mouzouris and J. M. Mendel, "Nonsingleton fuzzy logic systems: theory and application," IEEE Trans. Fuzzy Syst., vol. 5, no. 1, pp. 56$71,1997$.

[6] J. M. Mendel, Uncertain rule-based fuzzy logic system: introduction and new directions. Prentice-Hall, 2001.

[7] C. Wagner and H. Hagras, "Novel methods for the design of general type-2 fuzzy sets based on device characteristics and linguistic labels surveys," in Proceedings of the Joint 2009 International Fuzzy Systems Association World Congress and 2009 European Society of Fuzzy Logic and Technology Conference. European Society for Fuzzy Logic and Technology, 2009, pp. 537-543.

[8] A. B. Cara, I. Rojas, H. Pomares, C. Wagner, and H. Hagras, "On comparing non-singleton type-1 and singleton type-2 fuzzy controllers for a nonlinear servo system," in IEEE Symposium on Advances in Type2 Fuzzy Logic Systems. IEEE, 2011, pp. 126-133.

[9] G. C. Mouzouris and J. M. Mendel, "Non-singleton fuzzy logic systems," in Fuzzy Systems, 1994. IEEE World Congress on Computational Intelligence., Proceedings of the Third IEEE Conference on. IEEE, 1994, pp. 456-461.

[10] N. Sahab and H. Hagras, "A type-2 nonsingleton type-2 fuzzy logic system to handle linguistic and numerical uncertainties in real world environments," in IEEE Symposium on Advances in Type-2 Fuzzy Logic Systems (T2FUZZ). IEEE, 2011, pp. 110-117.

[11] A. Pourabdollah, C. Wagner, and J. Aladi, "Improved uncertainty capture for non-singleton fuzzy systems," (accepted for) IEEE Transaction on Fuzzy Systems, 2015.

[12] A. Pourabdollah, C. Wagner, and J. Aladi, "Changes under the hood-a new type of non-singleton fuzzy logic system," in Fuzzy Systems (FUZZIEEE), 2015 IEEE International Conference on. IEEE, 2015, pp. 1-8.

[13] E. H. Mamdani and S. Assilian, "An experiment in linguistic synthesis with a fuzzy logic controller," International journal of man-machine studies, vol. 7, no. 1, pp. 1-13, 1975.

[14] L. Wang, A course in fuzzy systems. Prentice-Hall press, USA, 1999.

[15] R. Zwick, E. Carlstein, and D. V. Budescu, "Measures of similarity among fuzzy concepts: A comparative analysis," International Journal of Approximate Reasoning, vol. 1, no. 2, pp. 221-242, 1987.

[16] V. V. Cross and T. A. Sudkamp, Similarity and compatibility in fuzzy set theory: assessment and applications. Springer Science \& Business Media, 2002, vol. 93.

[17] P. Jaccard, Nouvelles recherches sur la distribution florale, 1908.

[18] I. Turksen and Z. Zhong, "An approximate analogical reasoning approach based on similarity measures," IEEE Trans. Syst., Man, Cybern., vol. 18, no. 6, pp. 1049-1056, 1988.

[19] D. S. Yeung and E. C. C. Tsang, "A comparative study on similaritybased fuzzy reasoning methods," IEEE Trans. Syst., Man, Cybern., vol. 27, no. 2, pp. 216-227, 1997.

[20] D.-G. Wang, Y.-P. Meng, and H.-X. Li, "A fuzzy similarity inference method for fuzzy reasoning," Computers \& Mathematics with Applications, vol. 56, no. 10, pp. 2445-2454, 2008.

[21] D. Yeung and E. Tsang, "Improved fuzzy knowledge representation and rule evaluation using fuzzy petri nets and degree of subsethood," International journal of intelligent systems, vol. 9, no. 12, pp. 10831100, 1994

[22] M. C. Mackey, L. Glass et al., "Oscillation and chaos in physiological control systems,” Science, vol. 197, no. 4300, pp. 287-289, 1977.

[23] L. Wang and J. M. Mendel, "Generating fuzzy rules by learning from examples," IEEE Trans. Syst., Man, Cybern., vol. 22, no. 6, pp. 1414 1427, 1992.

[24] J. McCulloch, C. Wagner, and U. Aickelin, "Analysing fuzzy sets through combining measures of similarity and distance," in 2014 IEEE International Conference on Fuzzy Systems. IEEE, 2014, pp. 155-162. 